\title{
Single Machine Scheduling Problem with Rejection to Minimize the Total Earliness plus the Total Rejection Cost
}

\author{
Ling Nie, Member, IACSIT
}

\begin{abstract}
In the classical scheduling, it is always assumed that for any job, it should be processed. However, in the real world, things may be more flexible and the decision makers can make a higher-level decision, i.e., they can break the constrain by rejecting a job. It's not hard for the readers to find examples in the industrial and commercial fields to justify this breaking. To reject a job, of course, the decision makers should pay a corresponding penalty. In this paper, we considered single-machine scheduling problems under the job rejection constraint. A job was either rejected, in which case a rejection penalty had to be paid, or accepted and processed on the single machine. In this paper, we considered fuzziness in the job scheduling problem, i.e., due-dates of all jobs are fuzzy variables. The objective was to minimize the sum of the maximum earliness of the accepted jobs and the total rejection penalty of the rejected jobs. We found a polynomial-time solution for the problem through finding the Pareto optimal points.
\end{abstract}

Index Terms-Rejected penalty, maximum earliness, Pareto optimal points, fuzzy due-date.

\section{INTRODUCTION}

The current increasingly competitive global marketplace has challenged the manufacturers to output products with low production cost through least possible utilization of resources, quick enough to deliver to customers on time and minimum congestion in the shop floor. Scheduling, a decision making process which deals with the allocation of limited resources to tasks over time plays an important role in achieving these goals.

In the scheduling problem with job rejection, we have a set of $\mathrm{n}$ jobs $\left\{J_{1}, \ldots, J_{n}\right\}$ and a single machine. Each job $J_{j}$ has a processing time $p_{j}>0$, a release date $r_{j}>0$ and a rejection penalty $e_{j}>0$. Job $J_{j}$ is either rejected, in which case a rejection penalty $e_{j}$ has to be paid, or accepted and processed (scheduled) on the machine. There are many research have been fund in this area, see [1]-[4].

In this scheduling research, the problems also related to earliness and tardiness are widely studied in the past decades. The topic is motivated by the Just-In-Time production which emphasizes the notion of earliness as well as tardiness. In a Just-In-Time scheduling environment, jobs that complete either earlier or later than due dates will be punished. Therefore, an ideal schedule is the one in which all jobs complete exactly at their due dates. There are many measures of performance among which the most common one is to minimize the deviation of job completion times around their due dates, see [5]-[10].

Manuscript received August 19, 2014; revised November 10, 2014.

Ling Nie is with Uncertainty Decision-Making Laboratory, Sichuan University, Chengdu 610064, China (e-mail: n1586512@163.com).
However, when formulating scheduling problems with rejection which closely describe and represent the real-world problems, various factors involved in the problems are often only imprecisely or ambiguously known to the analyst. This is particularly true in the real-world situations when human-centered factors are incorporated into the problems. In such situations, it may be more appropriate to consider fuzzy processing time due to man-made factors and fuzzy due-date tolerating a certain amount of delay in the due-date. To be more specific, considering a number of man-made factors that exist in operations and planning and other arrangements is also a part of operations, fuzziness in processing times undeniably exists. Concerning due-date, we can also imagine many situations where it is desirable in principle to strictly satisfy the due-date, a certain amount of delay may be tolerated with longer delay tends to be lower evaluations', see [11], [12].

Under these circumstances, in this paper, on the basis of the agreement index of fuzzy due-date and fuzzy processing time, we formulate a single machine scheduling problem with rejection to minimize the total earliness plus the total rejection cost.

\section{PROBLEM DESCRIPTION}

The problem considered in this paper can be stated as follows. There are $\mathrm{n}$ jobs which have to be processed on a single machine with no preemption. Let $J=J_{1}, J_{2}, \ldots, J_{n}$ be the job set. $p_{j}$ and $d_{j}$ are used to denote the processing time and the due date of job $J_{j}$, respectively, $j=1,2, \ldots, n$. Let $\alpha_{j}$ and $\beta_{j}$ be the earliness penalty and tardiness penalty per unit time of job $J_{j}$, respectively, $j=1,2, \ldots, n$. Given a schedule $\sigma$ for $J$, let $C_{j}(\sigma)$ be the completion time of job $J_{j}$ in $\sigma, j=1,2, \ldots, n$. Then the cost of schedule $\sigma$ is defined to be , where $E_{j}(\sigma)=\max \{0$, $\left.d_{j}-C_{j}(\sigma)\right\}$ is the earliness of job $J_{j}$ in and $T_{j}(\sigma)=\max \{0$, $\left.C_{j}(\sigma)-d_{j}\right\}$ is the tardiness of job $J_{j}$ in $\sigma, j=\{1,2, \ldots, n\}$. The objective is to find a schedule with the minimum cost. We follow the three-field notation to represent the problem by . In particular, we call the deviation of job $J_{j}$ in schedule $\sigma, j=1$, $2, \ldots, n$. Then is the total deviation of all jobs in $\sigma$.

Comparison with traditional scheduling problem, there are some uncertain factors in the actual scheduling problems. These uncertainty is mainly reflected in two aspects: the one is uncertain processing times, and the other uncertain due-date. In this paper, we consider a single-machine scheduling problem with fuzzy processing time to minimize the total earliness plus the total rejection cost.

In traditional machining operations, the processing time of a job is affected by the proficiency of workers, the operating status of the machine and other factors. And so we know the 
distribution of time only by experience and historical data. Even in the modern production line, the normal production processing time can be known while the preparation time is often still uncertain. Moreover, machine failures, maintenances and other factors still exist, making the overall machining time be still uncertain.

In addition, the traditional piece will be limited to a definite delivery date. But in the actual contract, the delivery time will be limited to a strict deadline when the time expressed. If this time is exceeded, it will have to pay a breakup fee. Thus, fuzzy variables to describe the due-date is often more appropriate.

In this paper, the processing times are often denoted by triangle fuzzy variables $P_{j}\left(p_{j}{ }^{1}, p_{j}{ }^{2}, p_{j}{ }^{3}\right.$ ) (see Fig. 1), and the due-dates are expressed by trapezoid fuzzy numbers $\left(d_{i}^{a}, d_{i}^{b}\right.$, $d_{i}^{c}, d_{i}{ }^{d}$ ) (see Fig. 2). In Fig. $1, p_{j}{ }^{1}$ and $p_{j}{ }^{3}$ are the lower bound and upper bound of the triangle fuzzy variable, respectively. $\mathrm{p}_{\mathrm{j}}{ }^{2}$ is the main value, and $u_{j}(x)$ is the membership. In Fig. 2, if the job is finished in the due-date time window $\left(d_{i}^{b}, d_{i}^{c}\right)$, then the satisfaction degree of this job is 1 , otherwise, the satisfaction degree will be depicted by a linear membership function $u_{i}(c)$, where $\mathrm{c}$ is the completion time of jobs. Submit your manuscript electronically for review.

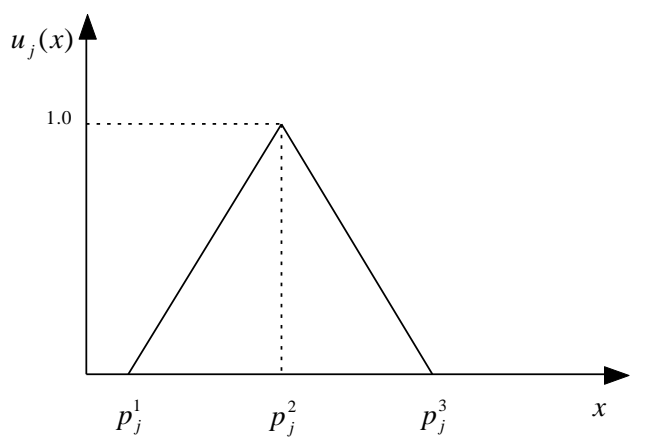

Fig. 1. Triangle fuzzy processing time.

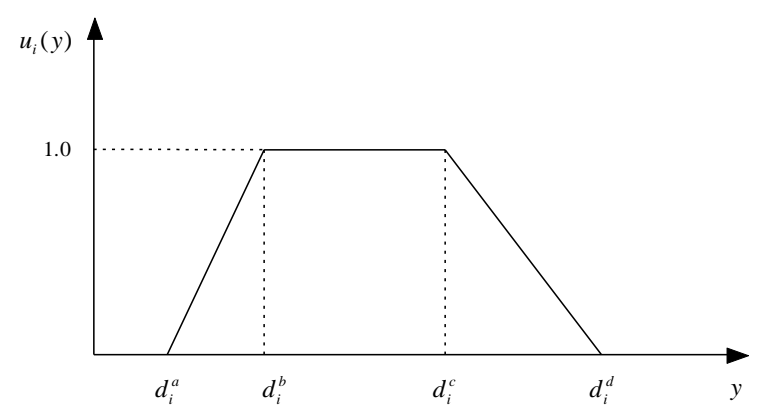

Fig. 2. Trapezoid fuzzy duedate.

\section{How to DEAL WITH FUZZY PROCESSING TIME AND PROCESSING DUEDATE}

In fuzzy case, fuzzy number (FNs) is often used to indicate processing conditions and some operations of FNs are essential to build a schedule. These operations are addition operation and max operation of two FNs as well as the ranking methods of FNs. Addition operation is often used to calculate the fuzzy completion time, max operation is used to determine the fuzzy beginning time and the ranking method is for the maximum fuzzy completion time, etc.

Denote a triangular fuzzy number $A$ by a triplet $\left(a_{1}, a_{2}\right.$, $\left.a_{3}\right)$. Then, as is well-known, the addition of two triangular fuzzy numbers $A=\left(a_{1}, a_{2}, a_{3}\right)$ and $B=\left(b_{1}, b_{2}, b_{3}\right)$ is shown by the following formula:

$$
\tilde{A}+\tilde{B}=\left(a_{1}, a_{2}, a_{3}\right)+\left(b_{1}, b_{2}, b_{3}\right)=\left(a_{1}+b_{1}, a_{2}+b_{2}, a_{3}+b_{3}\right) .
$$

The addition is used when calculating the fuzzy completion time of each operation.

Furthermore, denote the membership functions of two triangular fuzzy numbers $A=\left(a_{1}, a_{2}, a_{3}\right)$ and $B=\left(b_{1}, b_{2}, b_{3}\right)$ by $\mu_{A}$ and $\mu_{B}$, respectively, then according to the extension principle of Zadeh, the membership function $\mu_{A \vee B}(z)$ of $A \vee B$ through the $\vee(\max )$ operation becomes as follows:

$$
\mu_{A \vee B}(z)=\sup _{z=x \vee y} \min \left(\mu_{A}(z), \mu_{B}(z)\right)
$$

Unfortunately however, since the fuzzy number obtained as a result of the $\mathrm{V}(\max )$ operation through the extension principle does not always become a triangular fuzzy number, in this paper, for simplicity, we approximate the $\mathrm{V}(\max )$ operation with the following formula:

$$
\tilde{A} \vee \tilde{B}=\left(a_{1}, a_{2}, a_{3}\right) \vee\left(b_{1}, b_{2}, b_{3}\right) \cong\left(a_{1} \vee b_{1}, a_{2} \vee b_{2}, a_{3} \vee b_{3}\right)
$$

Here, although this formula is written with the approximating equal sign $\cong$, the varying relationships of the left and right spread of $A$ and $B$ could well result in cases where an equal sign and not just an approximation is established. Such situations are illustrated in Fig. 3. When $A$ and $B$ are in the positional relationships of (a) and (b), $A \vee B=B$ holds exactly. Although $A \vee B$ does not become a triangular fuzzy number in case of (c), we use the $\mathrm{V}(\max )$ operation shown above to approximate it as a triangular fuzzy number. Such $\mathrm{V}(\max )$ operation is used when calculating the fuzzy starting time for each operation.

The operation is used by Sakawa and Mori [13] and named Sakawa criterion for simplicity. Sakawa criterion has been extensively applied to build a complete scheduling of the fuzzy problem. Lei [14] compared these two criteria extensively.

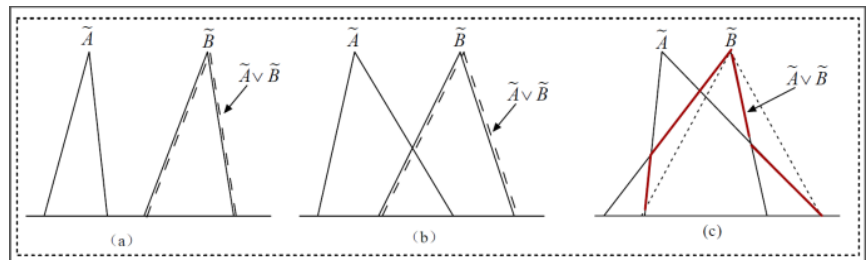

Fig. 3. V operation of triangular fuzzy numbers.

In this problem, fuzzy completion times becomes FNs, since they are calculated as the sum of fuzzy processing times. Hence, when considering to minimize the maximum fuzzy completion time as an objective function, some ranking methods become necessary for ranking the fuzzy completion times.

In this paper, we adopt the following ranking method for 
FNs. In this ranking method, the criterion for dominance is one of the following three in the order given below.

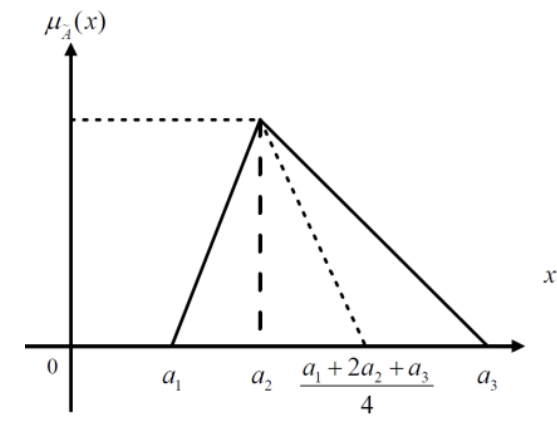

Fig. 4. The greatest associate ordinary number of $A$.

Criterion 1: If $\mathrm{C}_{1}(A)=\left[\left(a_{1}+2 a_{2}+a_{3}\right) / 4\right]>$ $(<) \mathrm{C}_{1}(B)=\left[\left(b_{1}+2 b_{2}+b_{3}\right) / 4\right]$, then $A>(<) B$;

Criterion 2: If $\mathrm{C}_{1}(A)=\mathrm{C}_{1}(B)$, then $\mathrm{C}_{2}(A)=a_{2}$ is compared with $\mathrm{C}_{2}(B)=b_{2}$ to rank them;

Criterion 3: If they have the identical $C_{1}$ and $C_{2}$, the difference of spreads $\mathrm{C}_{3}(A)=a_{3}-a_{1}$ is chosen as a third criterion.

According to these three criteria, it becomes possible to rank all FNs. Among FNs $A_{\mathrm{i}}, i=1,2, \ldots, n$, the maximum and minimum FNs are respectively denoted by $A_{\max }$ and $A_{\min }$. For example, according to these three criteria, determine the order of FNs of $S=\left\{A_{1}, A_{2}, A_{3}, A_{4}\right\}$, where $A_{1}=(2,5,8), A_{2}=(3,4,9), A_{3}=(3,5,7), A_{4}=(4,5,8)$.

Using the first criterion $\mathrm{C}_{1}, \mathrm{C}_{1}\left(A_{4}\right)=5.5$ and $\mathrm{C}_{1}\left(A_{1}\right)=\mathrm{C}_{1}\left(A_{2}\right)=\mathrm{C}_{1}\left(A_{3}\right)=5.0$. Hence $A_{\max }=A_{4}$. By the second criterion $\mathrm{C}_{2}, \mathrm{C}_{2}\left(A_{1}\right)=\mathrm{C}_{2}\left(A_{3}\right)=5$ and $\mathrm{C}_{2}\left(A_{2}\right)=4$. Thus $A_{\min }=A_{2}$. The third criterion $\mathrm{C}_{3}$ shows $\mathrm{C}_{3}\left(A_{1}\right)=6$ and $\mathrm{C}_{3}\left(A_{3}\right)=4$. In this way, the decreasing order of FNs of $S$ becomes $A_{4}, A_{1}, A_{3}, A_{2}$.

\section{AlgORITHM}

We know that the scheduling problem $1 \| E_{\max }$ is a ordinary problem when there is idle time on the machine. Set $d_{\max }=\max _{j} d_{j}, P=\sum_{j=1}^{n} p_{j}, s_{j}=d_{j}-p_{j}$, then the scheduling problem $1|\mathrm{nmit}| E_{\max }$ can be solved by STST rule, where STST rule means all jobs will be arranged by non decreasing sequence according to $s_{j}$.

In the actual production, the warehouses for all factories have limited capacity. If the orders are finished too fast, the backlog will be happened and then holistic benefit will be infected. Therefore, the decision makers can assign some orders to other producers but need to pay a certain processing fees which is the so-called rejection costs. For the scheduling problem $1|\mathrm{nmit}| E_{\max }+\sum_{J i} \in_{R} w_{j}$, there is a optimal sequence that all received jobs will be arranged by non decreasing sequence according to $s_{j}$.

Lemma 1. For the problem $1|\mathrm{nmit}| E_{\max }+\sum_{J_{j} \in \bar{S}} e_{j}$, there is an optimal schedule that all received jobs will arranged by STST rule.
According to Lemma 1, we sort the indexes of all jobs that $s_{1} \leq s_{2} \leq \ldots \leq s_{n}$. According to the above analysis, all off the Pareto optimal points for the scheduling problem $1|\mathrm{nmit}| E_{\max }+\sum_{J_{j} \in \bar{S}} e_{j}$ can be found by the following algorithm:

Set $N=\left\{J_{1}, \ldots, J_{n}\right\}$.

Step 1: Set $S:=N, \bar{S}=\varnothing$;

Step 2: Calculate $E_{\max }{ }^{1}=\max _{J j} \in S{ }^{\mathrm{STST}}\left\{E_{j}\right\}$;

Step 3: Denote $\mathrm{T}=\left\{J_{k}: E_{k}=E_{\max }{ }^{1}, J_{k} \in S\right\}$;

Step 4: Set $S^{\prime}:=S / T$, calculate $E_{\max }{ }^{2}=\max _{J j} \in S^{\prime}{ }^{\mathrm{STST}}\left\{E_{j}\right\}$;

Step 5: If $E_{\max }{ }^{2}<E_{\max }^{1}$, then $S:=S^{\prime}, \bar{S}:=\bar{S} \cup \mathrm{T}$, print out $\left(E_{\max }, \sum_{J_{j} \in \bar{S}} e_{j}\right)$ turn to step 2;

Step 6: If $E_{\max }\left(S^{\prime}\right) \geq E_{\max }(S)$, then shop, print out $S$ and $\bar{S}$. Set the above points set to be $H$, then $|H| \leq n$. Set the Pareto optimal points set of the scheduling problem $1|\mathrm{nmit}| E_{\max }+\sum_{J_{j} \in \bar{S}} e_{j}$ be $L$, then $L \subseteq H$, thus $|L| \leq$

$n$. Meanwhile, there are at most $\mathrm{n}$ iterations in the above algorithm, and each iteration will take a intrinsic time. Thus, the run time is polynomial and the value of the objective function is $\min \{f(x)+g(y):(x, y) \in H\}$.

Based on the analysis of the eto optimal points of the scheduling problem $1 \mid$ nmit $\mid E_{\max }+\sum_{J_{j} \in \bar{S}} e_{j}$, we can conclude the following algorithm:

Step 1: Set $S:=N, \bar{S}=\varnothing$;

Step 2: Calculate $E_{\max }(S)=\max _{J j} \in S\left\{E_{j}\right\}$;

Step 3: Denote $T=\left\{J_{k}: E_{k}=E_{\max }, J_{k} \in S\right\}$;

Step 4: Set $S^{\prime}:=S \backslash T$, calculate $E_{\max }\left(S^{\prime}\right)$;

Step 5: If $E_{\max }\left(S^{\prime}\right)+\sum_{J_{j} \in T \cup \bar{S}} e_{j}<E_{\max }(S)$, then $S:=$

$S^{\prime}, \bar{S}:=\bar{S} \cup T$, turn to step 2 ;

Step 6: If $E_{\max }\left(S^{\prime}\right)+\sum_{J_{j} \in T \cup \bar{S}} e_{j} \geq E_{\max }(S)$, then shop, print out $S$ and $\bar{S}$.

Therefore, the value of the objective function is $E_{\max }+\sum_{J_{j} \in \bar{S}} e_{j}$ and the run time of this algorithm is $O\left(n^{2}\right)$.

\section{CONCLUSION}

In this paper, we considered single-machine scheduling problems under the job rejection constraint. A job was either rejected, in which case a rejection penalty had to be paid, or accepted and processed on the single machine. In this paper, we considered fuzziness in the job scheduling problem, i.e., due-dates of all jobs are fuzzy variables. The objective was to minimize the sum of the maximum earliness of the accepted jobs and the total rejection penalty of the rejected jobs. We found a polynomial-time solution for the problem through finding the Pareto optimal points. 
This paper presents the state of the art and the research method of the fuzzy scheduling problems, and discusses the fuzzy operation about the processing time and the due-date as well as the fuzzy scheduling system based on rejection. At last, we gives an algorithm to find out the solution of a single machine scheduling problem with rejection to minimize the total earliness plus the total rejection cost under fuzzy processing time and fuzzy due-date constraints.

Future research plans to extend this model to multiple machines and/or job-shop scheduling problems as this study only discussed a single machine scheduling problem. Moreover, considering the multi-agent single systems to solve the problem using different programming method under different uncertain environments is as well as a future research direction.

\section{REFERENCES}

[1] P. Brucker, A. Gladky, H. Hoogeveen, M. Y. Kovalyov, C. N. Potts, and S. L. van de Velde, "Scheduling a batching machine," Journal of scheduling, vol. 1, pp. 31-54, 1998.

[2] Y. Bartal, S. Leonardi, A. M. Spaccamela, J. Sgall, and L. Stougie, "Multiprocessor scheduling with rejection," SIAM Journal on Discrete Mathematics, vol. 13, pp. 64-78, 2000.

[3] D. W. Engels, D. R. Karger, S. G. Kolliopoulos, S. Sengupta, R. N. Uma, and J. Wein, "Techniques for scheduling with rejection," Journal of Algorithms, vol. 49, pp.175-191, 2003.

[4] H. Hoogeveen, "Multicriteria scheduling," European Journal of Operational Research, vol. 167, pp. 592-623, 2005.

[5] J. A. Hoogeveen and S. L. van de Velde, "Scheduling with target start times," European Journal of Operational Research, vol. 129, pp. $87-94,2001$.

[6] R. L. Graham,E. L. Lawer, J. K. Lenstra, and A. H. G. Rinnooy Kan, "Optimization and ap-proximation in deterministic sequencing and scheduling: A survey," Annals of Discrete Math-Ematics, vol. 5, pp. $1-15,1979$.

[7] K. R. Baker and G. D. Scudder, "Sequencing with earliness and tardiness penalities: a review," Operations Research, vol. 38, pp. 22-36, 1990.

[8] J. B. Sidney, "Optimal single-machine scheduling with earliness and tardiness penalties," Operations Research, vol. 25, pp. 62-69, 1977.

[9] J. A. Ventura and S. Radhakrishnan, "Single machine scheduling with symmetric earliness and tardiness penalties," European Journal of Operational Research, vol. 144, pp. 598-612, 2003.

[10] L. Wan and J. Yuan, "Single-machine scheduling to minimize the total earliness and tardiness is strongly NP-hard," Operations Research Letters, vol. 41, pp. 363-365, 2013.

[11] H. Ishii and M. Tada, "Single machine scheduling problem with fuzzy precedence relation," European Journal of Operational Research, vol. 87, pp. 284-288, 1995

[12] Y. Tsujimura, M. Gen, and E. Kubota, "Solving job-shop scheduling problem with fuzzy processing time using genetic algorithm," Journal of Japan Society for Fuzzy Theory and Systems, vol. 7, no. 5, pp. 1073-1083, 1995

[13] M. Sakawa and T. Mori, "An efficient genetic algorithm for job shop scheduling problems with fuzzy processing time and fuzzy due date," Computers and Industrial Engineering, vol. 36, pp. 325-341, 1999.

[14] D. M. Lei, "Fuzzy job shop scheduling problem with availability constraints," Computers and Industrial Engineering, vol. 58, pp. 610-617, 2010.

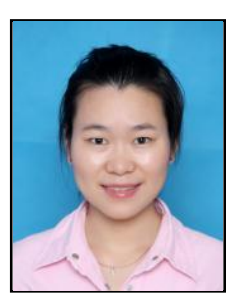

Ling Nie was born in 1987. She received her B.S. and M.S. degrees in Zhengzhou University. She is currently a Ph. D. at Sichuan University. Her research interests include uncertain decision making and scheduling with maintenance. 